\title{
Universal quantum gate with hybrid qubits in circuit quantum electrodynamics
}

\author{
Chui-Ping Yang ${ }^{1} *$ Zhen-Fei Zheng ${ }^{2}$, and Yu Zhang ${ }^{3}$ \\ ${ }^{1}$ Department of Physics, Hangzhou Normal University, Hangzhou 310036, China \\ ${ }^{2}$ Key Laboratory of Quantum Information, \\ University of Science and Technology of China, Heifei 230026, China and \\ ${ }^{3}$ School of Physics, Nanjing University, Nanjing 210093, China
}

\begin{abstract}
Hybrid qubits have recently drawn intensive attention in quantum computing. We here propose a method to implement a universal controlled-phase gate of two hybrid qubits via two threedimensional (3D) microwave cavities coupled to a superconducting flux qutrit. For the gate considered here, the control qubit is a microwave photonic qubit (particle-like qubit), whose two logic states are encoded by the vacuum state and the single-photon state of a cavity, while the target qubit is a cat-state qubit (wave-like qubit), whose two logic states are encoded by the two orthogonal cat states of the other cavity. During the gate operation, the qutrit remains in the ground state; therefore decoherence from the qutrit is greatly suppressed. The gate realization is quite simple, because only a single basic operation is employed and neither classical pulse nor measurement is used. Our numerical simulations demonstrate that with current circuit QED technology, this gate can be realized with a high fidelity. The generality of this proposal allows to implement the proposed gate in a wide range of physical systems, such as two 1D or 3D microwave or optical cavities coupled to a natural or artificial three-level atom. Finally, this proposal can be applied to create a novel entangled state between a particle-like photonic qubit and a wave-like cat-state qubit.
\end{abstract}

PACS numbers: 03.67.Bg, 42.50.Dv, 85.25.Cp

*Electronic address: yangcp@hznu.edu.cn 
Quantum gates, acting on hybrid qubits (i.e., different types of qubits), have attracted tremendous attention, because of their importance in connecting quantum information processors with different encoding qubits as well as their significant application in transferring quantum states between a quantum processor and a quantum memory. In recent years, many theoretical proposals have been presented for realizing a universal two-qubit controlled-phase (CP) or controlled-not (CNOT) gate with various hybrid qubits, such as a cat-state qubit and a charge qubit [1], a flying photonic qubit and an atomic qubit [2], a charge qubit and an atomic qubit [3], a spin qubit and a Majorana qubit [4], a photonic qubit and a superconducting qubit [5], and so on. Moreover, the two-qubit CP or CNOT gate with a flying optical photon and a single trapped atom [6], as well as the two-qubit $\mathrm{CP}$ gate with a ${ }^{40} \mathrm{Ca}^{+}$ qubit and one ${ }^{43} \mathrm{Ca}^{+}$qubit [7] have been demonstrated in experiments.

Circuit QED, consisting of microwave cavities and superconducting (SC) qubits, has been considered as one of the most promising candidates for quantum computing $[8,9]$. Besides SC qubits, microwave photonic qubits (encoded in the photon-number states) and cat-state qubits (encoded in superposition of coherent states) are two types of important qubits for quantum information processing (QIP) and quantum communication. Particularly, cat-state qubits have drawn intensive attention due to their enhanced life times [10]. Recently, much progress has been made for quantum state engineering and QIP with microwave photonic qubits [11-14] or cat-state qubits [15-18].

The goal of this letter focuses on realizing a two-qubit CP gate with two hybrid qubits, i.e., a microwave photonic qubit and a cat-state qubit, based on a circuit-QED [Fig.1(a)]. The two-qubit CP gate considered here is described by

$$
\begin{aligned}
& |0\rangle|c a t\rangle \rightarrow|0\rangle|c a t\rangle,|0\rangle|\overline{c a t}\rangle \rightarrow|0\rangle|\overline{c a t}\rangle, \\
& |1\rangle|c a t\rangle \rightarrow|1\rangle|c a t\rangle,|1\rangle|\overline{c a t}\rangle \rightarrow-|1\rangle|\overline{c a t}\rangle,
\end{aligned}
$$

where $|c a t\rangle$ and $|\overline{c a t}\rangle$ are two orthogonal cat states, representing the two logic states of a cat-state qubit, while $|0\rangle$ and $|1\rangle$ are the two logic states of a microwave photonic qubit. Eq. (1) implies that when the control qubit (first qubit) is in the state $|1\rangle$, a phase flip happens to the state $|\overline{c a t}\rangle$ of the target qubit (second qubit). It is known that a two-qubit CP gate, together with single-qubit gates, forms a set of universal gates for quantum computing [19].

This proposal has the following advantages. During the gate operation, the coupler qutrit remains in the ground state and thus decoherence from the qutrit is greatly suppressed. 
The gate realization is quite simple because only one-step operation is needed. Moreover, neither classical pulse nor measurement is required. Our numerical simulation shows that high-fidelity implementation of the gate is feasible with the current circuit QED technology. This proposal can be extended to realize the proposed gate with two 1D or 3D microwave or optical cavities coupled to a natural or artificial three-level atom.

Note that a two-qubit CP gate can be easily transferred to a two-qubit CNOT gate, by performing a Hadamard gate on the target qubit before and after the two-qubit CP gate [19]. Therefore, our proposal can also be applied to realize a hybrid two-qubit CNOT gate, described by $|0\rangle|c a t\rangle \rightarrow|0\rangle|c a t\rangle,|0\rangle|\overline{c a t}\rangle \rightarrow|0\rangle|\overline{c a t}\rangle,|1\rangle|c a t\rangle \rightarrow|1\rangle|\overline{c a t}\rangle$, and $|1\rangle|\overline{c a t}\rangle \rightarrow$ $|1\rangle|c a t\rangle$, acting on the two hybrid qubits. To the best of our knowledge, how to realize a two-qubit $\mathrm{CP}$ or CNOT gate with a microwave photonic qubit and a cat-state qubit has not been reported yet.

The two-qubit CP or CNOT gate here allows to create a novel entangled state $|0\rangle \mid$ cat $\rangle+$ $|1\rangle|\overline{c a t}\rangle$, through first preparing a microwave photonic qubit in the state $|0\rangle+|1\rangle$ while a cat-state qubit in the state $|c a t\rangle$ and then applying the above-mentioned two-qubit CNOT gate. This type of entangled state provides the first test of a Bell inequality violation between a particle-like photonic qubit and a wave-like cat-state qubit and has applications in hybrid quantum communication. Recently, hybrid entanglement $|0\rangle|\alpha\rangle+|1\rangle|-\alpha\rangle$ between a particle-like photonic qubit (encoded with $|0\rangle$ and $|1\rangle$ ) and a wave-like coherent-state qubit (encoded with the coherent states $|\alpha\rangle$ and $|-\alpha\rangle$ ) or between quantum and classical states of light [20,21] has been demonstrated in experiments, which has drawn increasing attention because hybrid entanglement of light is a key resource in establishing hybrid quantum networks and connecting quantum processors with different encoding qubits.

Consider two 3D microwave cavities inductively coupled to a SC flux qutrit [Fig. 1(a)]. The qutrit has three levels $|g\rangle,|e\rangle$ and $|f\rangle$ [Fig. 1(b)]. The $|g\rangle \leftrightarrow|e\rangle$ transition is weak due to the barrier between the two potential wells. Cavity 1 is dispersively coupled to the $|g\rangle \leftrightarrow|f\rangle$ transition with coupling constant $g_{1}$ and detuning $\delta_{1}$ but highly detuned (decoupled) from the $|e\rangle \leftrightarrow|f\rangle$ transition of the qutrit. In addition, cavity 2 is dispersively coupled to the $|e\rangle \leftrightarrow|f\rangle$ transition with coupling constant $g_{2}$ and detuning $\delta_{2}$ but highly detuned (decoupled) from the $|g\rangle \leftrightarrow|f\rangle$ transition of the qutrit (Fig. 2). These conditions can be met by prior adjustment of the qutrit's level spacings or the cavity frequency. For a $\mathrm{SC}$ qutrit, the level spacings can be rapidly (within 1-3 ns) tuned [22]. The frequency of a 


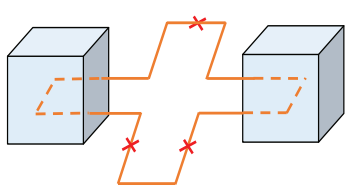

(a)

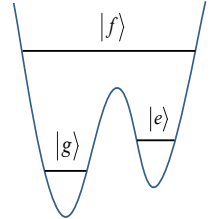

(b)

FIG. 1: (Color online) (a) Diagram of two 3D cavities inductively coupled to a superconducting flux qutrit. The qutrit consists of three Josephson junctions and a superconducting loop. (b) Level configuration of the flux qutrit, for which the transition between the two lowest levels can be made weak by increasing the barrier between two potential wells.

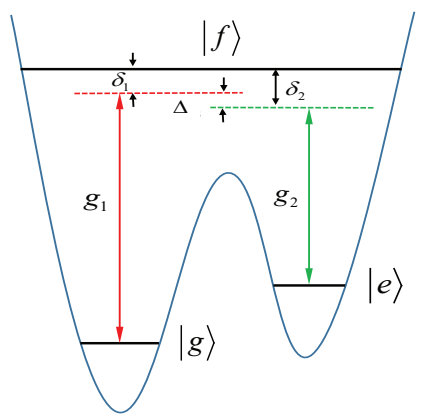

FIG. 2: (Color online) Cavity 1 is dispersively coupled to the $|g\rangle \leftrightarrow|f\rangle$ transition of the qutrit with coupling strength $g_{1}$ and detuning $\delta_{1}$, while cavity 2 is dispersively coupled to the $|e\rangle \leftrightarrow|f\rangle$ transition of the qutrit with coupling strength $g_{2}$ and detuning $\delta_{2}$. The red vertical line represents the frequency $\omega_{c_{1}}$ of cavity 1 , while the blue vertical line represents the frequency $\omega_{c_{2}}$ of cavity 2 . microwave cavity can be rapidly adjusted with a few nanoseconds [23].

Under the above assumptions, the Hamiltonian of the whole system in the interaction picture and after the rotating-wave approximation, is given by (in units of $\hbar=1$ )

$$
H_{\mathrm{I}}=g_{1}\left(e^{-i \delta_{1} t} \hat{a}_{1}^{+} \sigma_{f g}^{-}+\text {h.c. }\right)+g_{2}\left(e^{-i \delta_{2} t} \hat{a}_{2}^{+} \sigma_{f e}^{-}+\text {h.c. }\right),
$$

where $\hat{a}_{1}\left(\hat{a}_{2}\right)$ is the photon annihilation operator of cavity $1(2), \sigma_{f g}^{-}=|g\rangle\left\langle f\left|, \sigma_{f e}^{-}=\right| e\right\rangle\langle f|$, $\delta_{1}=\omega_{f g}-\omega_{c_{1}}>0$, and $\delta_{2}=\omega_{f e}-\omega_{c_{2}}>0$ (Fig. 2). Here, $\omega_{c_{1}}\left(\omega_{c_{2}}\right)$ is the frequency of cavity 1 (2); while $\omega_{f g}, \omega_{f e}$, and $\omega_{e g}$ are the $|f\rangle \leftrightarrow|g\rangle,|f\rangle \leftrightarrow|e\rangle$, and $|e\rangle \leftrightarrow|g\rangle$ transition frequencies of the qutrit, respectively.

By applying the large-detuning conditions $\delta_{1} \gg g_{1}$ and $\delta_{2} \gg g_{2}$, the Hamiltonian (2) can 
be written as $[24]$

$$
\begin{aligned}
H_{\mathrm{e}}= & -\lambda_{1} \hat{n}_{1}|g\rangle\left\langle g\left|-\lambda_{2} \hat{n}_{2}\right| e\right\rangle\left\langle e\left|+\left(\lambda_{1}+\lambda_{2}+\lambda_{1} \hat{n}_{1}+\lambda_{2} \hat{n}_{2}\right)\right| f\right\rangle\langle f| \\
& -\lambda\left(e^{i \triangle t} \hat{a}_{1}^{+} \hat{a}_{2} \sigma_{e g}^{-}+\text {h.c. }\right),
\end{aligned}
$$

where $\sigma_{e g}^{-}=|g\rangle\langle e|, \lambda_{1}=g_{1}^{2} / \delta_{1}, \lambda_{2}=g_{2}^{2} / \delta_{2}, \lambda=\left(g_{1} g_{2} / 2\right)\left(1 / \delta_{1}+1 / \delta_{2}\right), \triangle=\delta_{2}-\delta_{1}=$ $\omega_{c_{1}}-\omega_{c_{2}}-\omega_{e g}$ (Fig. 2); $\hat{n}_{1}=\hat{a}_{1}^{+} \hat{a}_{1}$ and $\hat{n}_{2}=\hat{a}_{2}^{+} \hat{a}_{2}$ are the photon number operators for cavities 1 and 2, respectively. The terms in the last line of Eq. (3) describe the $|e\rangle \leftrightarrow|g\rangle$ coupling induced by the two-cavity cooperation. For $\triangle \gg\left\{\lambda_{1}, \lambda_{2}, \lambda\right\}$, the Hamiltonian (3) becomes [24]

$$
\begin{aligned}
H_{\mathrm{e}} & =-\lambda_{1} \hat{n}_{1}|g\rangle\left\langle g\left|-\lambda_{2} \hat{n}_{2}\right| e\right\rangle\left\langle e\left|+\left(\lambda_{1}+\lambda_{2}+\lambda_{1} \hat{n}_{1}+\lambda_{2} \hat{n}_{2}\right)\right| f\right\rangle\langle f| \\
& -\chi \hat{n}_{1}\left(1+\hat{n}_{2}\right)|g\rangle\left\langle g\left|+\chi\left(1+\hat{n}_{1}\right) \hat{n}_{2}\right| e\right\rangle\langle e|
\end{aligned}
$$

where $\chi=\lambda^{2} / \Delta$. When the levels $|e\rangle$ and $|f\rangle$ are initially not occupied, they will remain unpopulated because the Hamiltonian (4) does not induce both $|g\rangle \rightarrow|e\rangle$ and $|g\rangle \rightarrow|f\rangle$ transitions. Hence, this Hamiltonian (4) reduces to

$$
H_{\mathrm{e}}=-\lambda_{1} \hat{n}_{1}|g\rangle\left\langle g\left|-\chi \hat{n}_{1}\left(1+\hat{n}_{2}\right)\right| g\right\rangle\langle g| \text {. }
$$

Assume that the qutrit is initially in the ground state $|g\rangle$. It will remain in this state because the Hamiltonian (5) cannot induce any transition for the qutrit. Therefore, the Hamiltonian (5) reduces to

$$
\widetilde{H}_{\mathrm{e}}=-\eta \hat{n}_{1}-\chi \hat{n}_{1} \hat{n}_{2}
$$

where $\eta=\lambda_{1}+\chi$. Here, $\widetilde{H}_{\mathrm{e}}$ is the effective Hamiltonian governing the dynamics of the two cavities. The unitary operator $U=e^{-i \widetilde{H}_{\mathrm{e}} t}$ is expressed as

$$
U=\exp \left[i \eta \hat{n}_{1} t\right] \otimes \exp \left(i \chi \hat{n}_{1} \hat{n}_{2} t\right)
$$

Let us now consider two hybrid qubits 1 and 2. Qubit 1 is a microwave photonic qubit (particle-like qubit), whose two logic states are represented by the vacuum state $|0\rangle$ and the single-photon state $|1\rangle$ of cavity 1. Qubit 2 is a cat-state qubit (wave-like qubit), whose two logic states are represented by the two orthogonal cat states $|c a t\rangle=M_{\alpha}^{+}(|\alpha\rangle+|-\alpha\rangle)$ and $|\overline{c a t}\rangle=M_{\alpha}^{-}(|\alpha\rangle-|-\alpha\rangle)$. Here, $M_{\alpha}^{ \pm}=1 / \sqrt{2\left(1 \pm e^{\left.-2|\alpha|^{2}\right)}\right.}$ are normalization coefficients. In terms of $|\alpha\rangle=e^{-|\alpha|^{2} / 2} \sum_{n=0}^{\infty} \frac{\alpha^{n}}{\sqrt{n !}}|n\rangle$ and $|-\alpha\rangle=e^{-|\alpha|^{2} / 2} \sum_{n=0}^{\infty} \frac{(-\alpha)^{n}}{\sqrt{n !}}|n\rangle$, we have

$$
|c a t\rangle=\sum_{m=0}^{\infty} C_{2 m}|2 m\rangle, \quad|\overline{c a t}\rangle=\sum_{n=0}^{\infty} C_{2 n+1}|2 n+1\rangle,
$$


where $C_{2 m}=2 M_{\alpha}^{+} e^{-|\alpha|^{2} / 2} \alpha^{2 m} / \sqrt{(2 m) !}$ and $C_{2 n+1}=2 M_{\alpha}^{-} e^{-|\alpha|^{2} / 2} \alpha^{2 n+1} / \sqrt{(2 n+1) !}$. Here, $m$ and $n$ are non-negative integers. From Eq. (8), one can see that the state $|c a t\rangle$ is orthogonal to the state $|\overline{c a t}\rangle$, which is independent of $\alpha$ (except for $\alpha=0$ ).

Based on Eq. (7) and Eq. (8), one can easily see that the unitary operator $U$ leads to the following state transformation

$$
\begin{aligned}
& |0\rangle_{1}|c a t\rangle_{2} \stackrel{U}{\longrightarrow}|0\rangle_{1}|c a t\rangle_{2} \\
& |0\rangle_{1}|\overline{c a t}\rangle_{2} \stackrel{U}{\longrightarrow}|0\rangle_{1}|\overline{c a t}\rangle_{2} \\
& |1\rangle_{1}|c a t\rangle_{2} \stackrel{U}{\longrightarrow} \sum_{m=0}^{\infty} \exp [i \eta t] \exp [i 2 m \chi t] C_{2 m}|1\rangle_{1}|2 m\rangle_{2}, \\
& |1\rangle_{1}|\overline{c a t}\rangle_{2} \stackrel{U}{\longrightarrow} \sum_{n=0}^{\infty} \exp [i \eta t] \exp [i(2 n+1) \chi t] C_{2 n+1}|1\rangle_{1}|2 n+1\rangle_{2},
\end{aligned}
$$

where subscripts 1 and 2 represents qubits 1 and 2 . For $\chi t=\pi$ and $\eta t=2 k \pi$ ( $k$ is a positive integer), Eq. (9) becomes

$$
\begin{aligned}
& |0\rangle_{1}|c a t\rangle_{2} \stackrel{U}{\longrightarrow}|0\rangle_{1}|c a t\rangle_{2} \\
& |0\rangle_{1}|\overline{c a t}\rangle_{2} \stackrel{U}{\longrightarrow}|0\rangle_{1}|\overline{c a t}\rangle_{2} \\
& |1\rangle_{1}|c a t\rangle_{2} \stackrel{U}{\longrightarrow}|1\rangle_{1}|c a t\rangle_{2}, \\
& |1\rangle_{1}|\overline{c a t}\rangle_{2} \stackrel{U}{\longrightarrow}-|1\rangle_{1}|\overline{c a t}\rangle_{2},
\end{aligned}
$$

which shows that when the control qubit 1 is in the state $|1\rangle$, a phase flip (from sign + to -) happens to the state $|\overline{c a t}\rangle$ of the target qubit 2. The state transformation (10) shows that a two-qubit CP gate, described by Eq. (1), is implemented by the above operation.

For the two-qubit controlled phase gate described in Eq. (1) or Eq. (10), the control qubit and the target qubit can exchange their functions. Namely, when the control qubit is a cat-state qubit and the target qubit is a microwave photonic qubit, the two-qubit controlled phase gate can still be realized with the above operation.

From the above description, it can be seen that the coupler qutrit remains in the ground state $|g\rangle$ during the entire operation. Hence, decoherence from the qutrit is greatly suppressed. In addition, the gate is realized with a single basic operation described by the unitary operator $U$. 


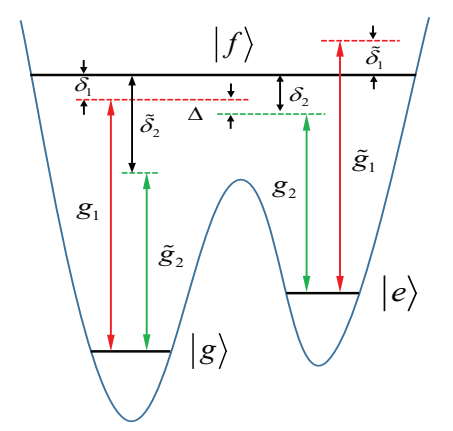

FIG. 3: (Color online) Illustration of the unwanted coupling between cavity 1 and the $|e\rangle \leftrightarrow|f\rangle$ transition of the qutrit (with coupling strength $\widetilde{g}_{1}$ and detuning $\widetilde{\delta}_{1}$ ) as well as the unwanted coupling between cavity 2 and the $|g\rangle \leftrightarrow|f\rangle$ transition of the qutrit (with coupling strength $\widetilde{g}_{2}$ and detuning $\left.\widetilde{\delta}_{2}\right)$. Note that the coupling of each cavity with the $|g\rangle \leftrightarrow|e\rangle$ transition of the qutrit is negligible because of the weak $|g\rangle \leftrightarrow|e\rangle$ transition.

In above, we have set $\chi t=\pi$ and $\eta t=2 k \pi$, resulting in

$$
g_{2}=\frac{2 \delta_{2}}{\delta_{1}+\delta_{2}} \sqrt{\frac{\delta_{1} \Delta}{2 k-1}} .
$$

In practice, the coupling strength $g_{2}$ can be adjusted by a prior design of the sample with appropriate capacitance or inductance between the qutrit and cavity 2 .

We now discuss the experimental feasibility of realizing the gate. In reality, there exist the unwanted coupling of cavity 1 with the $|e\rangle \leftrightarrow|f\rangle$ transition and the unwanted coupling of cavity 2 with the $|g\rangle \leftrightarrow|f\rangle$ transition of the qutrit (Fig. 3). After considering these factors, the Hamiltonian (2) is modified as

$$
\widetilde{H}_{\mathrm{I}}=H_{\mathrm{I}}+\delta H
$$

with

$$
\begin{aligned}
\delta H= & \widetilde{g}_{1}\left(e^{-i \widetilde{\delta}_{1} t} \hat{a}_{1}^{+} \sigma_{f e}^{-}+h . c .\right) \\
& +\widetilde{g}_{2}\left(e^{-i \widetilde{\delta}_{2} t} \hat{a}_{2}^{+} \sigma_{f g}^{-}+h . c .\right) .
\end{aligned}
$$

Here, $H_{\mathrm{I}}$ is the Hamiltonian $(2) ; \delta H$ is the Hamiltonian, which describes the unwanted coupling between cavity 1 and the $|e\rangle \leftrightarrow|f\rangle$ transition with coupling strength $\widetilde{g}_{1}$ and detuning $\widetilde{\delta}_{1}=\omega_{f e}-\omega_{c_{1}}$, as well as the unwanted coupling between cavity 2 and the $|g\rangle \leftrightarrow|f\rangle$ transition with coupling strength $\widetilde{g}_{2}$ and detuning $\widetilde{\delta}_{2}=\omega_{f g}-\omega_{c_{2}}$ (Fig. 3). 
When the dissipation and dephasing are included, the dynamics of the lossy system is determined by

$$
\begin{aligned}
\frac{d \rho}{d t}= & -i\left[\widetilde{H}_{\mathrm{I}}, \rho\right]+\sum_{l=1}^{2} \kappa_{l} \mathcal{L}\left[a_{l}\right] \\
& +\gamma_{e g} \mathcal{L}\left[\sigma_{e g}^{-}\right]+\gamma_{f e} \mathcal{L}\left[\sigma_{f e}^{-}\right]+\gamma_{f g} \mathcal{L}\left[\sigma_{f g}^{-}\right] \\
& +\sum_{j=e, f}\left\{\gamma_{\varphi j}\left(\sigma_{j j} \rho \sigma_{j j}-\sigma_{j j} \rho / 2-\rho \sigma_{j j} / 2\right)\right\}
\end{aligned}
$$

where $\widetilde{H}_{\mathrm{I}}$ is the above full Hamiltonian, $\sigma_{j j}=|j\rangle\langle j|(j=e, f)$, and $\mathcal{L}[\xi]=$ $\xi \rho \xi^{\dagger}-\xi^{\dagger} \xi \rho / 2-\rho \xi^{\dagger} \xi / 2$, with $\xi=a_{l}, \sigma_{e g}^{-}, \sigma_{f e}^{-}, \sigma_{f g}^{-}$. In addition, $\kappa_{l}$ is the photon decay rate of cavity $l(l=1,2), \gamma_{e g}$ is the energy relaxation rate for the level $|e\rangle$ of the qutrit, $\gamma_{f e}\left(\gamma_{f g}\right)$ is the energy relaxation rate of the level $|f\rangle$ of the qutrit for the decay path $|f\rangle \longrightarrow|e\rangle(|g\rangle)$, and $\gamma_{\varphi j}$ is the dephasing rate of the level $|j\rangle(j=e, f)$ of the qutrit.

For simplicity, we consider the two qubits are initially in the following state

$$
\begin{aligned}
\left|\psi_{\text {in }}\right\rangle= & \cos \alpha \cos \beta|0\rangle_{1}|c a t\rangle_{2}+\cos \alpha \sin \beta|0\rangle_{1}|\overline{c a t}\rangle_{2} \\
& +\sin \alpha \cos \beta|1\rangle_{1}|c a t\rangle_{2}+\sin \alpha \sin \beta|1\rangle_{1}|\overline{c a t}\rangle_{2} .
\end{aligned}
$$

Thus, the ideal output state of the whole system is

$$
\begin{aligned}
\left|\psi_{\text {id }}\right\rangle= & \left(\cos \alpha \cos \beta|0\rangle_{1}|c a t\rangle_{2}+\cos \alpha \sin \beta|0\rangle_{1}|\overline{c a t}\rangle_{2}\right. \\
& \left.+\sin \alpha \cos \beta|1\rangle_{1}|c a t\rangle_{2}-\sin \alpha \sin \beta|1\rangle_{1}|\overline{c a t}\rangle_{2}\right) \otimes|g\rangle .
\end{aligned}
$$

The fidelity of the operation is defined as

$$
\mathcal{F}=\frac{1}{(2 \pi)^{2}} \int_{0}^{2 \pi} \int_{0}^{2 \pi} \sqrt{\left\langle\psi_{\text {id }}|\rho| \psi_{\text {id }}\right\rangle} d \alpha d \beta,
$$

where $\left|\psi_{\text {id }}\right\rangle$ is the output state of an ideal system given above, without dissipation and dephasing; while $\rho$ is the final practical density operator of the system when the operation is performed in a realistic situation.

For a flux qutrit, the typical transition frequency between neighboring levels can be made as 1 to $20 \mathrm{GHz}$. As an example, consider $\omega_{e g} / 2 \pi=5.0 \mathrm{GHz}, \omega_{f e} / 2 \pi=7.5 \mathrm{GHz}$, and $\omega_{f g} / 2 \pi=12.5 \mathrm{GHz}$. With a choice of $\delta_{1} / 2 \pi=1.5 \mathrm{GHz}$ and $\delta_{2} / 2 \pi=1.65 \mathrm{GHz}$, 


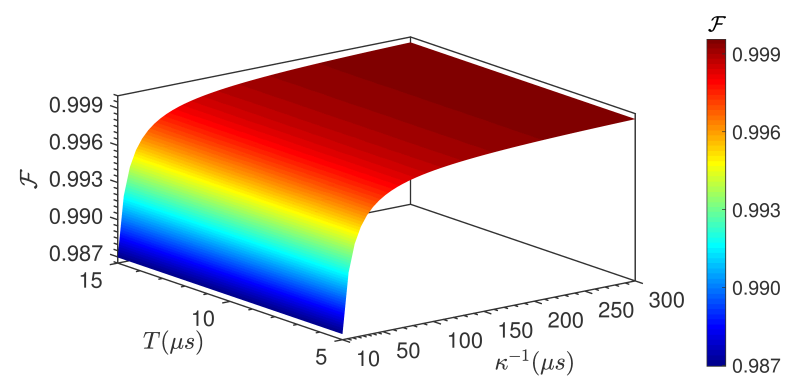

FIG. 4: (Color online) Fidelity versus $T$ and $\kappa^{-1}$. The parameters used in the numerical simulation are referred to the text.

we have $\Delta / 2 \pi=150 \mathrm{MHz}, \omega_{c_{1}} / 2 \pi=11 \mathrm{GHz}$, and $\omega_{c_{2}} / 2 \pi=5.85 \mathrm{GHz}$. For the transition frequencies of the qutrit and the frequencies of the cavities given here, we have $\widetilde{\delta}_{1} / 2 \pi=-3.5$ $\mathrm{GHz}$ and $\widetilde{\delta}_{2} / 2 \pi=6.65 \mathrm{GHz}$. Additional parameters used in the numerical simulation are: (i) $\gamma_{e g}^{-1}=5 T \mu \mathrm{s}, \gamma_{f e}^{-1}=2 T \mu \mathrm{s}, \gamma_{f g}^{-1}=T \mu \mathrm{s}$, (ii) $\gamma_{\phi e}^{-1}=\gamma_{\phi f}^{-1}=T \mu \mathrm{s}$, (iii) $\kappa_{1}=\kappa_{2}=\kappa$, (iv) $g_{1} / 2 \pi=150 \mathrm{MHz}$, and (v) $\alpha=0.5$. According to Eq. (11), a simple calculation gives $g_{2} / 2 \pi \sim 149.8 \mathrm{MHz}$. For a flux qutrit, $\widetilde{g}_{1} \sim g_{1}$ and $\widetilde{g}_{2} \sim g_{2}$. The coupling constants chosen here are readily available because a coupling constant $\sim 2 \pi \times 636 \mathrm{MHz}$ was reported for a flux device coupled to a microwave cavity [25].

By solving the master equation (14), we numerically plot Fig. 4, which illustrates the fidelity versus $T$ and $\kappa^{-1}$. From Fig. 4, one can see that when $T \geqslant 5 \mu \mathrm{s}$ and $\kappa^{-1} \geqslant 136 \mu \mathrm{s}$, fidelity exceeds $99.9 \%$, which implies that a high fidelity can be obtained for the gate being performed in a realistic situation.

With the parameters chosen above, the gate operational time is estimated as $\sim 0.37 \mu \mathrm{s}$, much shorter than the decoherence times of the qutrit $(5 \mu \mathrm{s}-75 \mu \mathrm{s})$ and the cavity decay times $(10 \mu \mathrm{s}-300 \mu \mathrm{s})$ considered in Fig. 4. In our numerical simulation, we consider a rather conservative case for decoherence time of the flux qutrit, because experiments have reported decoherence time $70 \mu \mathrm{s}$ to $1 \mathrm{~ms}$ for a superconducting flux device [26,27]. For $\kappa^{-1}=136 \mu \mathrm{s}$ and the cavity frequencies given above, a simple calculation gives $Q_{1} \sim 9.39 \times 10^{6}$ for cavity 1 and $Q_{2} \sim 4.99 \times 10^{6}$ for cavity 2 . Note that a high quality factor $Q=3.5 \times 10^{7}$ of a $3 \mathrm{D}$ microwave cavity has been experimentally demonstrated [18,28]. Our analysis here implies that the high-fidelity implementation of the proposed gate is feasible within the current circuit QED technology. 


\section{Funding Information}

This work was supported in part by the NKRDP of China (Grant No. 2016YFA0301802)

and the National Natural Science Foundation of China under Grant Nos. [11074062, $11374083,11774076]$.

[1] O. P. de SáNeto, and M. C. de Oliveira, J. Phys. B At. Mol. Opt. Phys. 45, 185505 (2012).

[2] G. Y. Wang, Q. Liu, H. R. Wei, T. Li, Q. Ai, and F. G. Deng, Sci. Rep. 6, 24183 (2016).

[3] D. Yu, M. M. Valado, C. Hufnagel, L. C. Kwek, L. Amico, and R. Dumke, Phys. Rev. A 93, 042329 (2016).

[4] S. Hoffman, C. Schrade, J. Klinovaja, and D. Loss, Phys. Rev. B 94, 045316 (2016).

[5] D. Kim and K. Moon, arxiv: 1808.02865.

[6] A. Reiserer, N. Kalb, G. Rempe, and S. Ritter, Nature (London) 508, 237 (2014)

[7] C. J. Ballance, V. M. Schäfer, J. P. Home, D. J. Szwer, S. C. Webster, D. T. C. Allcock, N. M. Linke, T. P. Harty, D. P. L. Aude Craik, D. N. Stacey, A. M. Steane and D. M. Lucas, Nature (London) 528, 384 (2015)

[8] Z. L. Xiang, S. Ashhab, J. Q. You, and F. Nori, Rev. Mod. Phys. 85, 623 (2013).

[9] J. Q. You and F. Nori, Nature (London) 474, 589 (2011).

[10] N. Ofek, A. Petrenko, R. Heeres, P. Reinhold, Z. Leghtas, B. Vlastakis, Y. Liu, L. Frunzio, S. M. Girvin, L. Jiang, M. Mirrahimi, M. H. Devoret, and R. J. Schoelkopf, Nature (London) 536, 441 (2016).

[11] Y. X. Liu, L. F. Wei, and F. Nori, Europhys. Lett. 67, 941 (2004).

[12] M. Hua, M. J. Tao, and F. G. Deng, Phys. Rev. A 90, 18824 (2014).

[13] A. N. Korotkov, Phys. Rev. B 84, 014510 (2011).

[14] C. P. Yang, Q. P. Su, S. B. Zheng, and S. Han, Phys. Rev. A 87, 022320 (2013).

[15] M. Mirrahimi, Z. Leghtas, V. V. Albert, S. Touzard, R. J. Schoelkopf, L.Jiang, and M.H.Devoret, New J. Phys. 16, 045014 (2014).

[16] S. E. Nigg, Phys. Rev. A 89, 022340 (2014).

[17] C. P. Yang, Q. P. Su, S. B. Zheng, F. Nori, and S. Han, Phys. Rev. A 95, 052341 (2017).

[18] C. Wang, Y. Y. Gao, P. Reinhold, R. W. Heeres, N. Ofek, K. Chou, C. Axline, M. Reagor, J. 
Blumoff, K. M. Sliwa, L. Frunzio, S. M. Girvin, L. Jiang, M. Mirrahimi, M. H. Devoret, and R. J. Schoelkopf, Science 352, 1087 (2016).

[19] M. Nielsen and I. Chuang, Quantum Computation and Quantum Information (Cambridge Univ. Press, Cambridge, 2000).

[20] O. Morin, K. Huang, J. Liu, H. L. Jeannic, C. Fabre, and J. Laurat, Nat. Photonics 8, 570 (2014).

[21] H. Jeong, A. Zavatta, M. Kang, S. W. Lee, L. S. Costanzo, S. Grandi, T. C. Ralph, and M. Bellini, Nat. Photonics 8, 564 (2014).

[22] P. J. Leek, S. Filipp, P. Maurer, M. Baur, R. Bianchetti, J. M. Fink, M. Göppl, L. Steffen, and A. Wallraff, Phys. Rev. B 79, 180511 (2009).

[23] M. Sandberg, C. M. Wilson, F. Persson, T. Bauch, G. Johansson, V. Shumeiko, T. Duty, and P. Delsing, Appl. Phys. Lett. 92, 203501 (2008).

[24] D. F. James and J. Jerke, Can. J. Phys. 85, 625 (2007).

[25] T. Niemczyk, F. Deppe, H. Huebl, E. P. Menzel, F. Hocke, M. J. Schwarz, J. J. Garcia Ripoll, D. Zueco, T. Hümmer, E. Solano, A. Marx, and R. Gross, Nat. Phys. 6, 772 (2010).

[26] F. Yan, S. Gustavsson, A. Kamal, J. Birenbaum, A. P. Sears, D. Hover, T. J. Gudmundsen, J. L. Yoder, T. P. Orlando, J. Clarke, A. J. Kerman, and W. D. Oliver, Nat. Commun. 7, 12964 (2016).

[27] J. Q. You, X. Hu, S. Ashhab, and F. Nori, Phys. Rev. B 75, 140515(R) (2007).

[28] M. Reagor, W. Pfaff, C. Axline, R. W. Heeres, N. Ofek, K. Sliwa, E. Holland, C. Wang, J. Blumoff, K. Chou, M. J. Hatridge, L. Frunzio, M. H. Devoret, L. Jiang, and R. J. Schoelkopf, Phys. Rev. B 94, 014506 (2016). 